\title{
Towards acoustic condition monitoring for detection and characterisation of laser induced breakdown in a gas turbine laser ignition system
}

\begin{tabular}{|r|l|}
\hline Journal: & International Journal of Condition Monitoring \\
\hline Manuscript ID & IJCM-12-2017-TN-0131.R1 \\
\hline Manuscript Type: & Technical Note \\
\hline Complete List of Authors: & $\begin{array}{l}\text { Griffiths, Jonathan; University of Lincoln College of Science, School of } \\
\text { Engineering } \\
\text { Grebenik, Jarek; University of Lincoln College of Science, School of } \\
\text { Engineering } \\
\text { Kirk, Antony; University of Lincoln College of Science, School of } \\
\text { Engineering }\end{array}$ \\
\hline Keyword: & Condition monitoring, Lasers, Acoustic emission methods (AE) \\
\hline &
\end{tabular}


1 Towards acoustic condition monitoring for detection and

2 characterisation of laser induced breakdown in a gas turbine

3 laser ignition system

4

J. GRIFFITHS ${ }^{1 *}$, J. GREBENIK ${ }^{1}$, and A. KIRK ${ }^{1}$

6

$7 \quad{ }^{1}$ School of Engineering, University of Lincoln, Brayford Pool, Lincoln, LN6 7TS, United

8 Kingdom

9

10

Tel: +4401522837936

12 E-mail:JGriffiths@lincoln.ac.uk

13

14

15

\section{ABSTRACT:}

Acoustic detection and characterisation of laser induced breakdown is an attractive proposition in laser ignition systems in which condition monitoring is necessary but where optical access for monitoring purposes is impractical. Presented is a signal processing method based on wavelet decomposition for the non-invasive detection of acoustic emissions resulting from laser induced breakdown in an atmospheric pressure combustion test rig, representative of a single combustion chamber in a sub $15 \mathrm{MW}$ industrial gas turbine. The probability and consistency of laser induced breakdown is determined from the acoustic signal and used to characterize the operating conditions and identify abrupt and incipient or slowly developing faults.

\section{KEYWORDS:}

Lasers; Condition monitoring; Acoustic emission methods (AE) 
1 Laser ignition (LI) offers the potential to address the durability issues associated with conventional high energy electrical ignition systems by removing the ignition apparatus from the hot gas path [1,2]. LI typically utilizes tightly focussed visible to near infrared nanosecond pulsed laser radiation to induce optical breakdown in combustible mixtures via a multi-photon ionization process for the purpose of initiating combustion $[3,4]$. In this process, multi-photon ionization of atoms in the target medium results in the release of electrons which readily absorb more photons, thereby increasing their kinetic energy. Subsequent collision of these electrons with neighbouring electrons results in avalanche ionization and the generation of a plasma in the focal region.

Condition monitoring is essential to the development of an LI system for gas turbines. Unsatisfactory operation of the LI system may be indicative of an incipient or slowly developing fault such as damage to optical elements in the beam path or an abrupt fault such as failure of a light guide. Both of the aforementioned fault types would require the activation of an interlock which is capable of reliably reducing the beam strength to safe levels within eye protection delay times, as defined in IEC 60825-2 [5]. Due to the nature of the application and associated hardware, there is limited optical access to the combustion chamber, making the effective use of optical means of condition monitoring impractical. There is potential for use of acoustic emissions to monitor the LI process, as has been applied to laser peening in recent years, a process with many similarities to $L I[9,10]$. A component of the energy within the plasma generated as a result of the multi-photon ionization process manifests itself as an acoustic emission in the audible frequency range. This is the result of a shockwave which is formed and expands around the plasma. Initially, expansion of this shockwave is driven by the high temperature plasma, whose energy increases due to absorption of laser radiation during the laser pulse [6]. After the laser pulse ends the temperature of the plasma reduces, resulting in a deceleration in the expansion of the shockwave. From this point, the blast wave model of Jones can be employed to describe the propagation of the shockwave into the acoustic region $[7,8]$. 
1 The principal challenge in the development of an acoustic emission based condition monitoring system for detection and characterisation of laser induced breakdown concerns resolving the acoustic emissions of interest in the relatively noisy environment associated with engine operation [11]. Relatively little literature exists concerning detection of acoustic emissions that result from laser induced breakdown. Yaacob et al. proposed utilising changes in the refractive index of multimode optical fibres to detect acoustic emissions resulting from laser induced breakdown in isolation oil [12]. It was shown that this method produced a noise free signal which required no signal processing. The linear dependence of acoustic pressure on laser pulse energy for optical breakdown in water was characterized by Bulanov et al. using a hydrophone and acoustic receiver [13]. Krasnenko et al. studied the sound generation in the atmosphere resulting from optical breakdown in aerosol sprays using a microphone, with a view to its practical application as a diagnostic tool for the influence of atmospheric conditions on laser induced breakdown regimes [14]. Hosoya et al. studied the time responses of sound pressure generated by laser induced breakdown in air in an anechoic box [15]. The acoustic signals resembled impulse responses, characterised by a high peak pressures, and were found to be highly reproducible in terms of pulse width and peak pressure. This reproducibility was attributed to the consistency of the laser as a source. No literature exists regarding detection of acoustic emissions from optical breakdown events in noisy environments associated with engine operation which are typical in LI applications.

Proposed in this study is a new approach to the condition monitoring of an LI system in realistic, engine-like environments. Information regarding the probability and consistency of laser induced breakdown is discerned from associated acoustic emissions using wavelet decomposition; a signal processing methodology which is uniquely suited to the extraction of impulse-like features heavily masked by noise. The results of this investigation reveal significant potential for the practical application of acoustic-based condition monitoring techniques in LI systems.

\section{2. METHODOLOGY}


1 In this section, details of the experimental arrangement used to record acoustic

2 signals are given followed by a description of the signal processing technique used to

3 analyse the data.

4

\section{$5 \quad 2.1$ Experimental arrangement}

6 An atmospheric pressure combustion test rig was utilized in this study, which could

7 be fitted with a single combustor can from a range of Siemens industrial gas

8 turbines. For the purpose of this investigation, the rig was fitted with a combustion

9 can and pilot burner from a small to medium (that is, sub $15 \mathrm{MW}$ output power)

10 Siemens industrial gas turbine.

11

12 A laser ignition system was developed utilizing a Q-switched Nd:YAG TEM 00 laser

13 (Brilliant; Quantel, Ltd.) with an $\mathrm{M}^{2}$ of 1.84, pulse duration of $4 \mathrm{~ns}$ and operating at

$1410 \mathrm{~Hz}$ repetition rate and $1064 \mathrm{~nm}$ wavelength. The output pulse energy $\left(E_{p}\right)$ of the

15 laser source was limited from its maximum of $360 \mathrm{~mJ}$ to approximately $21 \mathrm{~mJ}$ using

16 by flashlamp/Q-switch delay time to avoid damage to optical elements in the

17 system. With the flashlamp/Q-switch delay kept constant, a polarization based

18 optical attenuator consisting of a $1 / 2$ wave plate and polarizing beam splitting cube

19 was used to control the laser pulse energy. The laser pulse energy for a given $1 / 2$ wave

20 plate rotation was measured (UP19K-VR; Gentec Electro-Optics, Inc.) and averaged

21 over 100 pulses. The experimental arrangement is shown in Figure 1. 


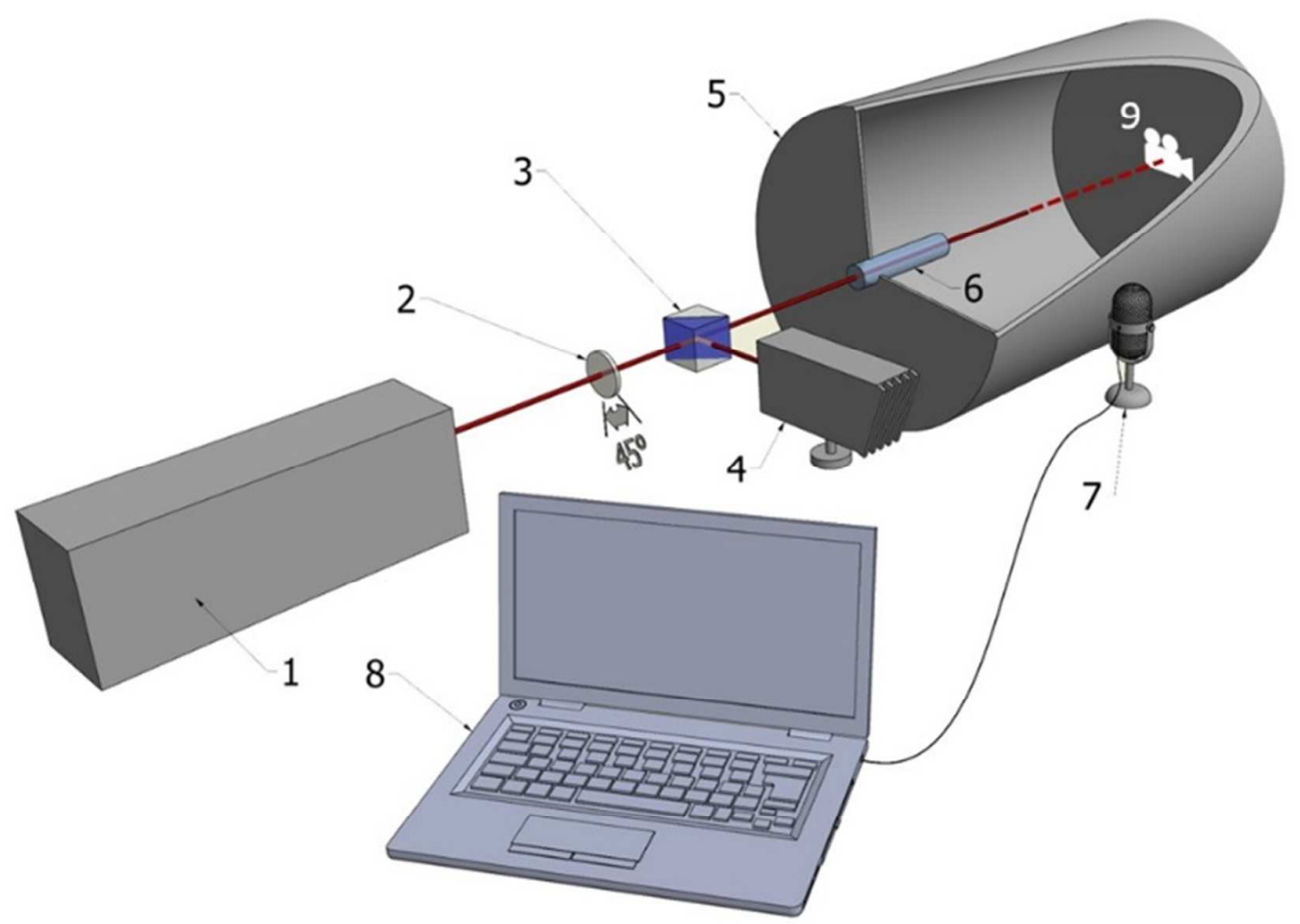

1

2

3

4

5

6

7

9

Figure 1: Experimental arrangement with 1) laser source, 2) $1 / 2$ wave plate, 3) polarizing beam splitting cube, 4) beam dump, 5) combustion chamber, 6) laser igniter assembly, 7) microphone, 8) data acquisition computer and 9) camera.

A custom laser igniter assembly was utilized, consisting of a clear aperture for transmission of the laser beam, a plano-convex focusing optic with an effective focal

8 length of $27 \mathrm{~mm}$ and an anti-reflective coated N-BK7 output window. The optical

9 path length from the laser source to the igniter assembly was approximately $3 \mathrm{~m}$.

0 The tip of the igniter assembly was sealed with red silicone around the edge of the 12

13 The presence or otherwise of laser induced breakdown within the combustion

14 chamber could be verified by monitoring the camera feed. Figure 2 shows the 15 camera feed for both flashlamp operation and laser induced breakdown. 

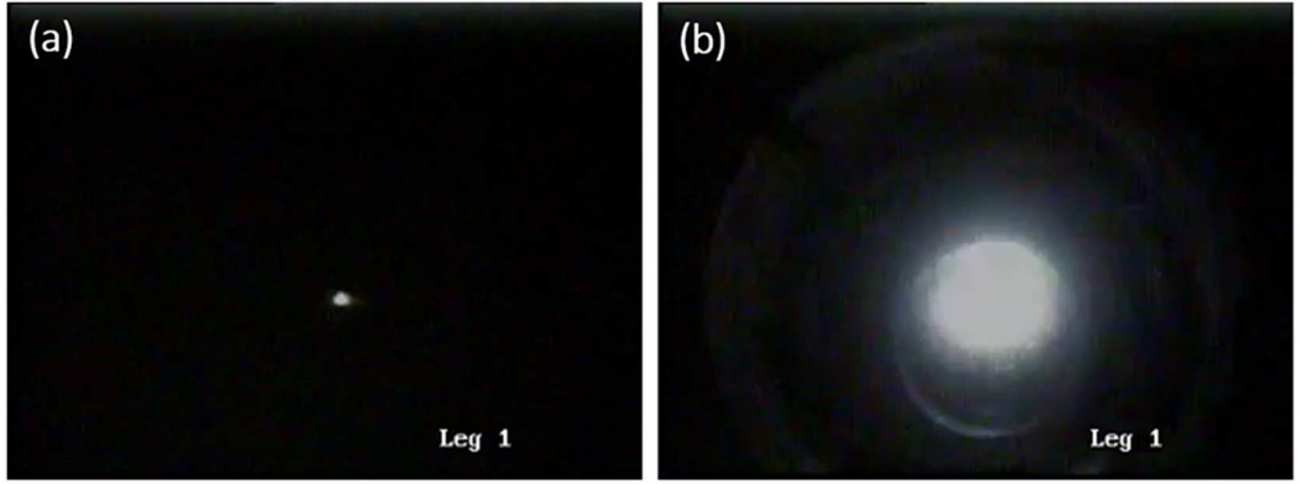

Figure 2: Camera feed showing (a) flashlamp operation and (b) laser induced breakdown $(10 \mathrm{~Hz}$ repetition rate, $1064 \mathrm{~nm}$ wavelength).

A condenser microphone (AT2020, Audio-Technica Ltd.) with a frequency response of 0.02 to $20 \mathrm{kHz}$ was used to capture the acoustic signal, which was sampled directly using MATLAB at $48 \mathrm{kHz}$ and 16-bit resolution. The sensor was placed approximately $2 \mathrm{~cm}$ from the combustion chamber's external casing with the sensitive plane aimed directly at the casing wall. In this investigation, the ambient conditions were such that no additional steps were required to reduce wind noise on the microphone.

\subsection{Signal processing methodology}

The signal processing work focused on acoustic identification of laser induced breakdown in an atmospheric pressure combustion test rig, representative of an industrial gas turbine during its start-up procedure. Audio recordings of $11 \mathrm{~s}$ in duration were repeated ten times for a given set of experiment parameters. The raw acoustic signal was sampled and imported directly into MATLAB. Each signal was clipped to a length of $10 \mathrm{~s}$ to remove unwanted artefacts from the beginning and end of the recording and then processed by two level wavelet decomposition, allowing for the resolution of information in both the time and frequency domains. Wavelet decomposition was chosen on the basis of both its sensitivity to abrupt changes in frequency in the time domain and its computational efficiency. The base wavelet best capable of resolving the laser induced breakdown events was selected using energy to Shannon entropy ratio at the first level of decomposition as the criterion [16]. A total of 131 base wavelet types from the wavelet families 
1 Daubechies, symlets, coiflets, biorthogonal, Fejer-Korovkin and reverse biorthogonal were considered, with the base wavelet producing the maximum energy to Shannon

3 entropy ratio selected as the most appropriate wavelet; in this case, the

4 biorthogonal (Bior6.8) wavelet. The wavelet decomposition produced three sets of coefficients, representing the approximation of the signal at low frequencies $\left(a_{1}\right)$ and the detail of the signal at high frequencies $\left(d_{1}\right.$ and $\left.d_{2}\right)$. Where present, laser induced breakdown events are clearly visible at the first level of decomposition in the detail coefficients $d_{1}$, as shown in Figure 3 for raw acoustic signal $s$, representing consistent laser induced breakdown in an atmospheric pressure combustion test rig. As such, the $d_{1}$ coefficients (henceforth referred to as the detail signal) were extracted for

11 further processing.
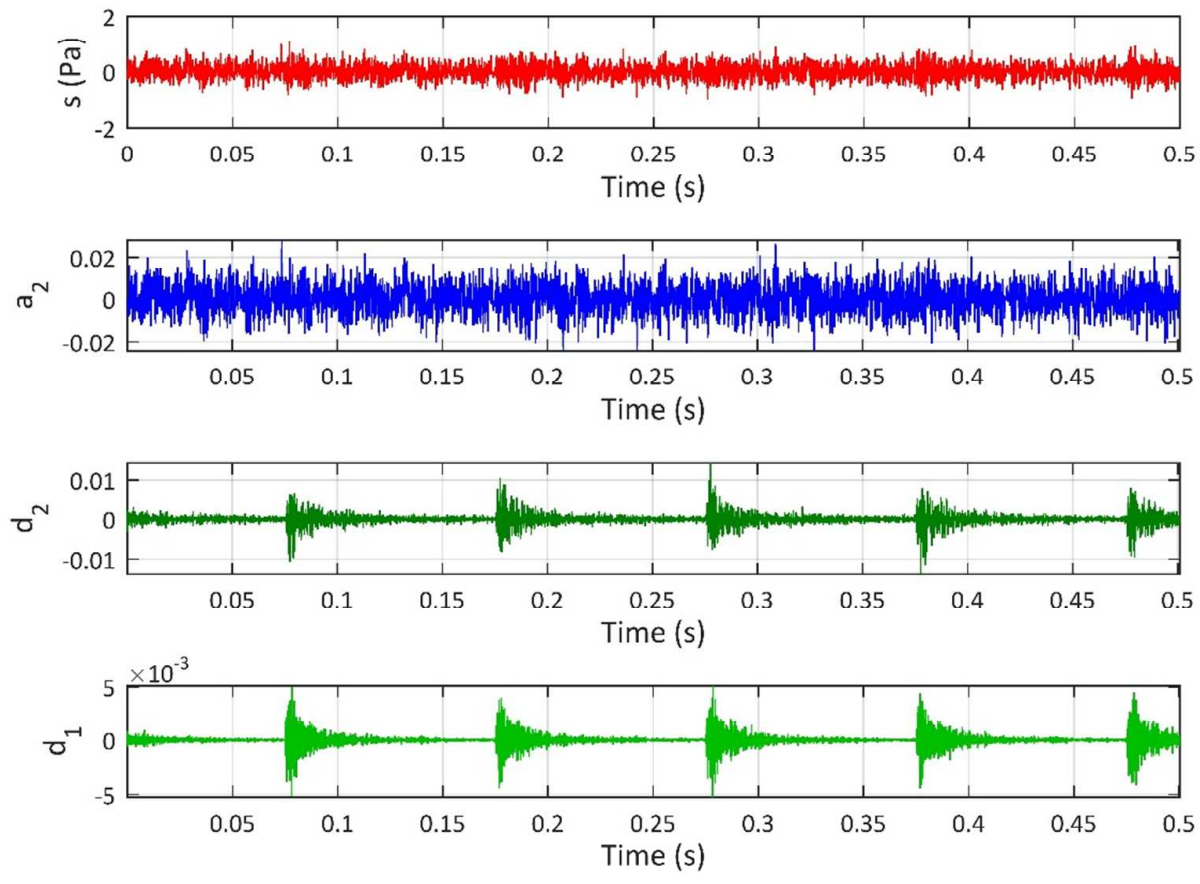

13

14

17

18

Figure 3: Two level Bior6.8 wavelet decomposition of raw acoustic sound pressure signal $s$ with approximation coefficients $a_{2}$, and detail coefficients $d_{1}$ and $d_{2}(21 \mathrm{~mJ}$ pulse energy, $10 \mathrm{~Hz}$ repetition rate, $1064 \mathrm{~nm}$ wavelength).

\section{RESULTS AND DISCUSSION}


J Griffiths et al.

1 The experimental work focused on (i) identification of abrupt faults and (ii)

2 identification of incipient or slowly developing faults in a gas turbine laser ignition

3 system. Acoustic signals were recorded during operation of the laser ignition system

4 on an atmospheric pressure combustion test rig with a constant air mass flow rate of

$5 \quad 0.4 \mathrm{~kg} / \mathrm{s}$. It should be noted that this intake of air into the combustion chamber was

6 the main source of noise when the test rig was in operation. Operation of intake and

7 exhaust fans also contributed to the noise. No fuel was introduced into the

8 combustion chamber in this investigation. This effectively recreates the conditions at

9 the point in the turbine start-up procedure at which the laser ignition system would

10 be fired for condition monitoring purposes. The acoustic signals were subsequently

11 processed according to the methodology outlined in Section 2.2. To simulate

12 unsatisfactory operation of the laser ignition system, the laser pulse energy $\left(E_{p}\right)$ was

13 increased from a minimum to the maximum pulse energy used in this investigation

14 of $21 \mathrm{~mJ}$ by rotating the $1 / 2$ wave plate in the experimental arrangement depicted in

15 Figure 1. A peak detection algorithm was applied to the detail signal to determine

16 the probability of laser induced breakdown as a function of laser pulses fired over a

17 given period of time, a representative output of which is shown in Figure 4. The

18 threshold above which the signal was considered to be a peak was dictated by the

19 maximum and minimum amplitude values for the detail signal at the first level of

20 decomposition for an ambient acoustic signal, recorded with test rig in operation

21 and the laser flashlamps on. The peak-to-peak separation was restricted using a

22 windowing function in accordance with the $10 \mathrm{~Hz}$ repetition rate of the laser source. 

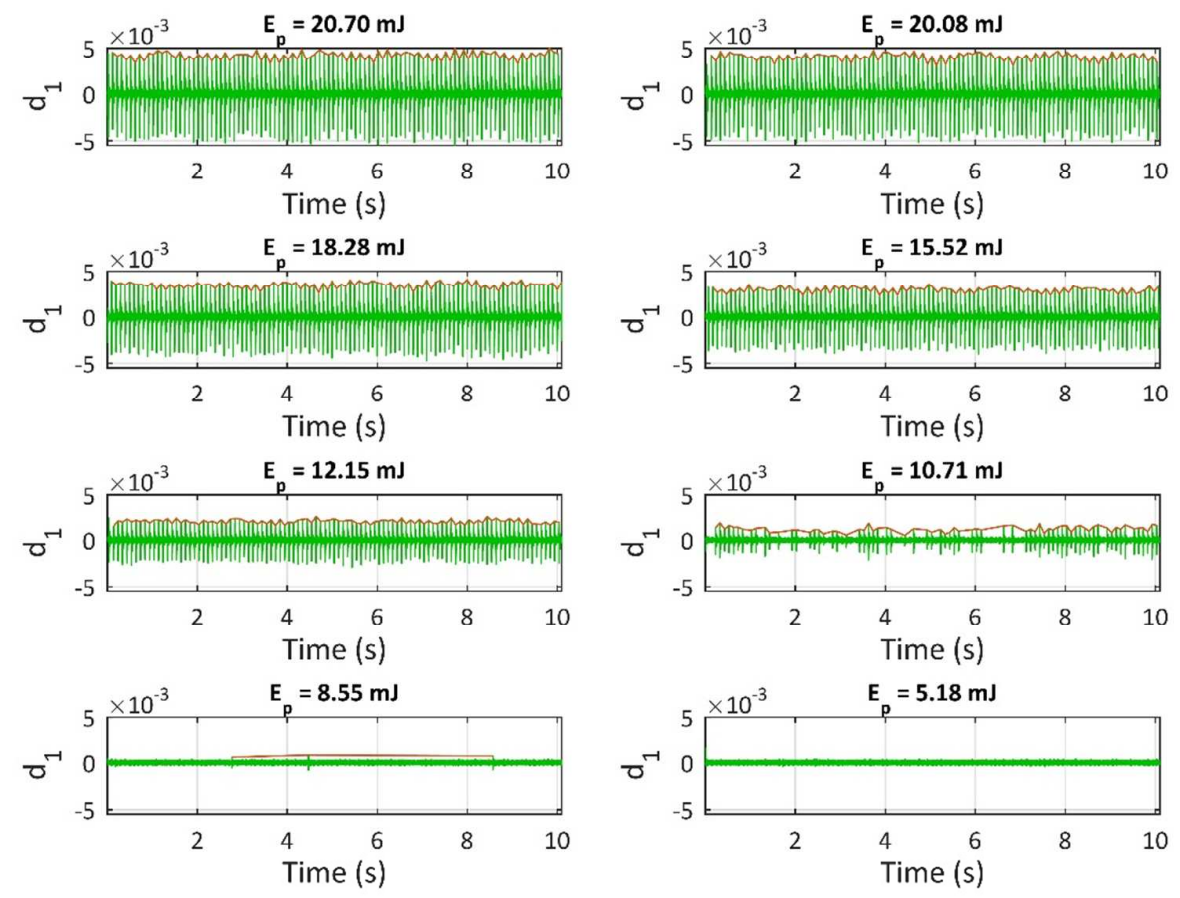

1

4

5

Figure 4: Representative outputs from the peak detection algorithm applied to the detail signal for a single $10 \mathrm{~s}$ audio recording (10 $\mathrm{Hz}$ repetition rate, $1064 \mathrm{~nm}$ wavelength).

Figure 4 reveals the presence of three distinct energy regions as the pulse energy is increased. At an incident pulse energy of $5.18 \mathrm{~mJ}$ no laser induced breakdown occurred. A second region was observed for incident pulse energies of $8.55 \mathrm{~mJ}$ and $10.71 \mathrm{~mJ}$, characterized by inconsistent laser induced breakdown with a probability of $2.7 \%$ and $64.6 \%$, respectively. Optical breakdown can only occur when the incident laser radiation is above a threshold value; therefore, for pulse energies close to this threshold value as opposed to those well in excess of it, a statistically smaller fraction of the laser pulse is capable of causing optical breakdown, resulting in a reduced probability of laser induced breakdown [17]. A third region of consistent laser induced breakdown was observed for incident pulse energies in excess of 12.15 $\mathrm{mJ}$, in accordance with observations made by Chen et. al [18]. The presence of these three distinct energy regions was confirmed by reviewing the recorded camera feed. 
1 In addition to the probability of laser induced breakdown, information relating to the

2 consistency of laser induced breakdown can be inferred from the detail signal. The

3 following sections detail methods for the identification of abrupt and incipient or

4 slowly developing faults from this information.

5

$6 \quad 3.1$ Abrupt fault detection

7 An abrupt fault would result in unsatisfactory operation of the laser ignition system

8 immediately after the fault develops. Possible causes for such a fault may include

9 misalignment, severe damage to an optical element in the beam path or failure of

10 the laser source to operate correctly. In the event of an abrupt fault, inconsistent or

11 no laser induced breakdown will occur. As such, the time interval between the peaks

12 detected in the detail signal is a useful parameter to consider. Figure 5 presents a

13 box plot of the peak intervals for those incident pulse energies which resulted in

14 laser induced breakdown.

(a)

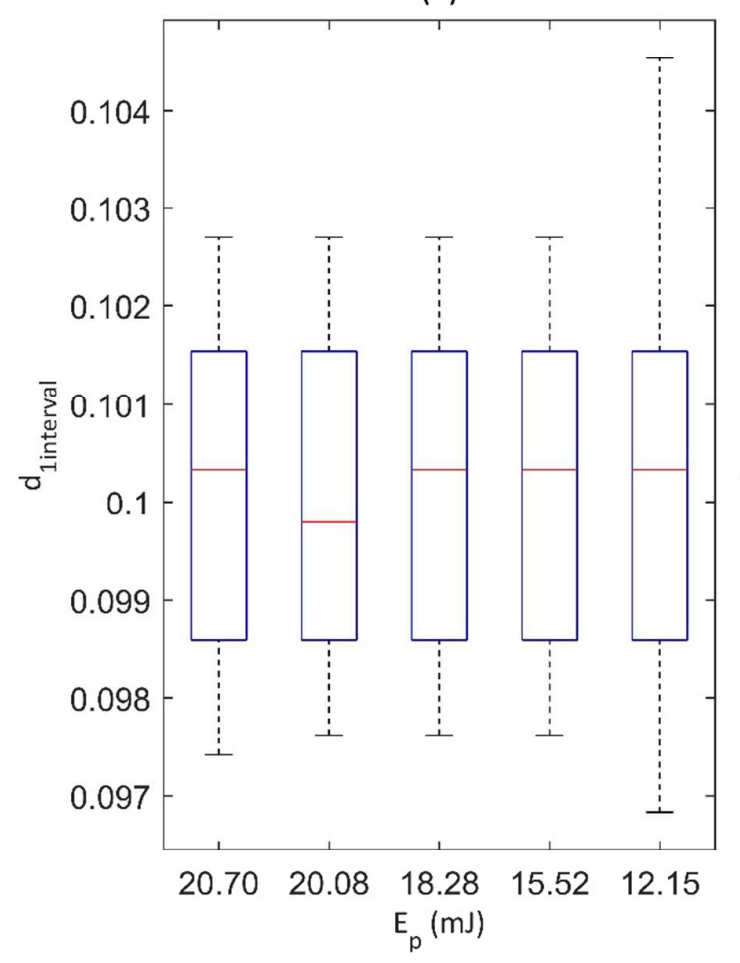

(b)

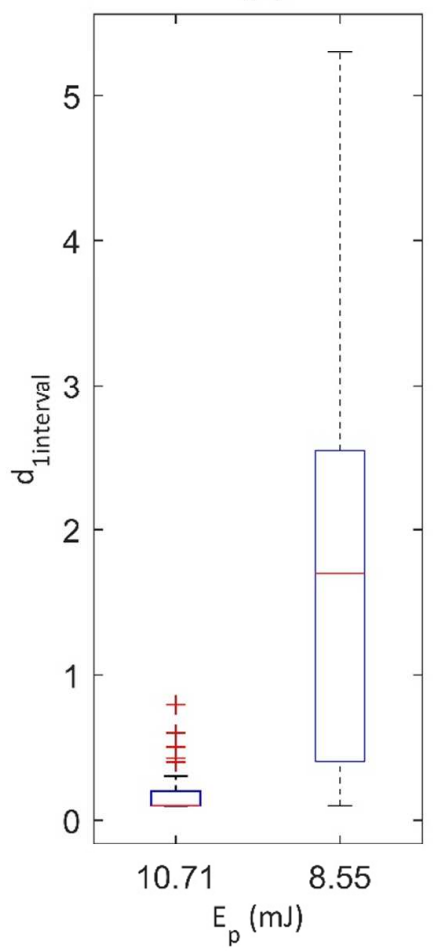

16

Figure 5: Box plot of the peak intervals $\left(d_{1 \text { interval }}\right)$ from the peak detection algorithm applied to the detail signals in (a) the consistent laser induced breakdown region and (b) the inconsistent laser induced breakdown region (10 $\mathrm{Hz}$ repetition rate, $1064 \mathrm{~nm}$ wavelength). 
2 Referring to Figure 5, for incident pulse energies in excess of $12.15 \mathrm{~mJ}$ (that is, in the

3 consistent laser induced breakdown region) the peak intervals are centred on $0.1 \mathrm{~s}$,

4 corresponding to the $10 \mathrm{~Hz}$ repetition rate of the laser source. As the incident pulse

5 energy decreases to $10.71 \mathrm{~mJ}$ and $8.55 \mathrm{~mJ}$ the median interval and distribution both

6 increase. This can be attributed to increasingly inconsistent spark formation as the

7 incident pulse energy decreases, therefore reducing the number of breakdown

8 events detected.

9

\section{$10 \quad 3.2$ Incipient fault detection}

11 An incipient or slowly developing fault may result in unsatisfactory operation of the 12 laser ignition system at some point after the fault initially develops. A possible cause

13 for such a fault could be slight damage to an optical element in the beam path,

14 acting as a nucleation point for further damage upon successive irradiation. In the 15 event of an incipient fault occurring, it is important to identify and rectify the 16 underlying issue as it can eventually lead to inconsistent laser induced breakdown 17 and therefore unsatisfactory operation of the laser ignition system. Figure 6 shows 18 the distribution of the absolute value of peak amplitudes as a function of incident 19 pulse energy. 


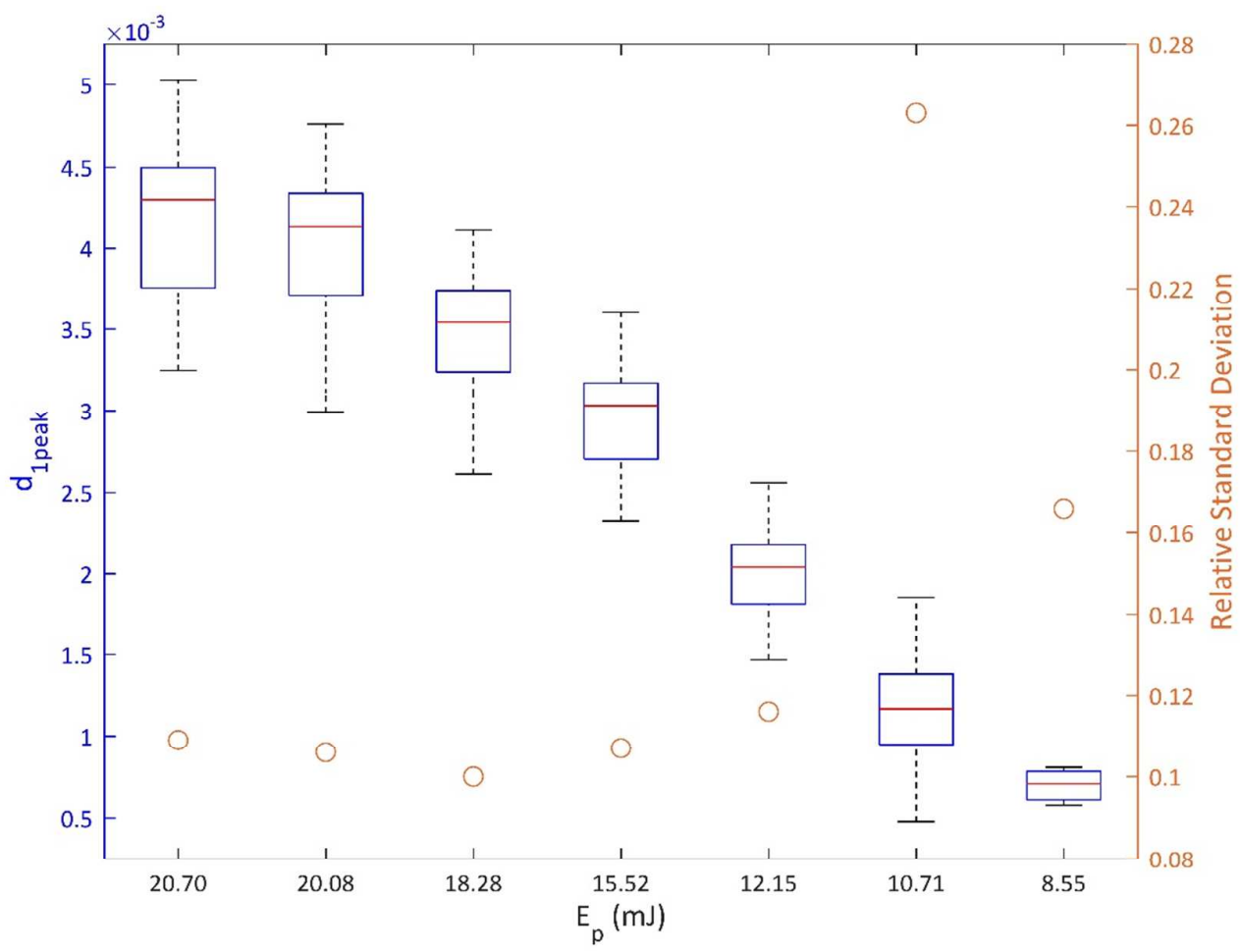

1

Figure 6: Box plot of absolute values of peak amplitude from the peak detection algorithm applied to the detail signal $\left(d_{1 \text { peak }}\right)$ and their relative standard deviation (circles) as a function of incident pulse energy ( $10 \mathrm{~Hz}$ repetition rate, $1064 \mathrm{~nm}$ wavelength).

Figure 6 reveals a decrease in the median absolute value of peak amplitude with decreasing incident pulse energy, following a sigmoidal trend. In the consistent laser induced breakdown region (that is, at incident pulse energies of $12.15 \mathrm{~mJ}$ and above) there is also a narrowing of the distribution with decreasing incident pulse energy. This narrowing can be attributed to the decrease in magnitude of the individual peak amplitudes as less energy is deposited in the shock wave. As such, this narrowing is not reflected in the relative standard deviation, which remains consistent in this region. The transition from consistent to inconsistent laser induced breakdown is characterized by an abrupt increase in the relative standard deviation at an incident pulse energy of $10.71 \mathrm{~mJ}$, corresponding to a breakdown probability of $64.6 \%$. Long term monitoring (that is, at every start-up sequence for the turbine) of the absolute value of peak amplitude could be used to monitor for the sigmoidal trend shown in Figure 6, indicative of an incipient or slowly developing fault. 


\section{CONCLUSIONS}

2 Noise robust acoustic condition monitoring for detection and characterisation of

3 laser induced breakdown in a laser ignition system has been demonstrated. Acoustic

4 detection of laser induced breakdown events under realistic engine-like conditions

5 was achieved through use of a signal processing method based on wavelet

6 decomposition using a Bior6.8 mother wavelet and extraction of the detail

7 coefficients at the first level of decomposition as a de-noised signal.

8

9 The potential for the implementation of an acoustic sensor system for condition monitoring of a gas turbine laser ignition system has been clearly demonstrated. A peak detection algorithm was applied to determine the probability of laser induced breakdown as a function of laser pulses fired over a given period of time. Information relating to the consistency of laser induced breakdown was then used to identify abrupt and incipient or slowly developing faults, simulated by decreasing the incident pulse energy. The time interval between peaks was shown to increase from a base of $0.1 \mathrm{~s}$ corresponding to the repetition rate of the laser as the energy region transitioned from consistent to inconsistent laser induced breakdown with decreasing incident pulse energy, indicative of an abrupt fault. The absolute value of peak amplitude was shown to decrease with decreasing incident pulse energy, following a sigmoidal trend which could be used to identify insipient faults. Further work is required to address the instrumentation and integration challenges associated with development of a real-time acoustic condition monitoring system for gas turbine laser ignition systems.

\section{ACKNOWLEDGEMENTS:}

The authors would like to thank Siemens Industrial Turbomachinery Ltd (SITL) for funding this investigation as part of a wider investigation into laser ignition for gas turbines.

\section{REFERENCES:}


[1] Griffiths J, Riley M, Kirk A, Borman A, Lawrence J, Dowding C. Lean burn limit and time to light characteristics of laser ignition in gas turbines. Optics and Lasers in Engineering 2014 4;55(0):262-266.

[2] Dearden G, Shenton T. Laser ignited engines: progress, challenges and prospects. Opt Express 2013;21:1113-1125.

[3] Tran X. P. Laser-induced spark ignition fundamental and applications. Optics and Lasers in Engineering 2006 5;44(5):351-397.

[4] Lorenz S, B \arwinkel M, Heinz P, Lehmann S, M\uhlbauer W, Br \uggemann D. Characterization of energy transfer for passively Q-switched laser ignition. Opt.Express 2015 Feb;23(3):2647-2659.

[5] Explosive atmospheres - Part 28: Protection of equipment and transmission systems using optical radiation. International Standard IEC 60079-28 2015; International Electrotechnical Commission.

[6] Gregorcic P, Diaci J, Mozina J. Two-dimensional measurements of laser-induced breakdown in air by high-speed two-frame shadowgraphy. Applied Physics A 2013 07/01;112(1):49-55.

[7] Jones DL. Intermediate Strength Blast Wave. The Physics of Fluids 1968 08/01; 2017/09;11(8):1664-1667.

[8] Gebel GC, Mosbach T, Meier W, Aigner M. Optical and spectroscopic diagnostics of laser-induced air breakdown and kerosene spray ignition. Combustion and Flame 2015 April 2015;162(4):1599-1613.

[9] Qiu C, Cheng L, He W. A novel acoustic monitoring method of laser peening. Appl Acoust 2016 9;110:9-12.

[10] Zhou L, Li Y, He W, Liu R, Chen L. Plasma Acoustic Wave Diagnostics in Laser Shock Processing. Lasers in Engineering 2014;28(1):45-56.

[11] Zimmer L, Tachibana S. Laser induced plasma spectroscopy for local equivalence ratio measurements in an oscillating combustion environment. Proceedings of the Combustion Institute 2007 January 2007;31(1):737-745.

[12] Yaacob MM, Alsaedi MA, Abdul Rahman R, Bidin N, Wajaht M, Nasir Al-geelani $A$, et al. Detection and wavelet analysis of acoustic emission signal from partial discharge captured by multimode optical fiber and piezoelectric sensors in insulation oil. International Journal of Physical Sciences 2013;8(21):1149-1160.

[13] Bulanov AV, Nagorny IG. Spectral and energy characteristics of acoustic emission in the laser radiation breakdown of water. Bulletin of the Russian Academy of Sciences: Physics 2014;78(2):160-163. 
1 [14] Krasnenko NP, Shamanaev SV. Sound generation in the atmosphere upon 2 exposure to high-power milli- and microsecond laser pulses. Russian Physics Journal 3 2009;52(9):944-956.

4 [15] Hosoya N, Nagata M, Kajiwara I. Acoustic testing in a very small space based on 5 a point sound source generated by laser-induced breakdown: Stabilization of plasma 6 formation. Journal of Sound and Vibration 201316 September 2013;332(19):4572-

74583.

8 [16] Yang Q, Wang J. Multi-Level Wavelet Shannon Entropy-Based Method for Single9 Sensor Fault Location. Entropy 2015;17:pp 7101-7117.

10 [17] Chen Y-, Lewis JWL, Parigger C. Spatial and temporal profiles of pulsed laser11 induced air plasma emissions. Journal of Quantitative Spectroscopy and Radiative 12 Transfer 2000 10/16;67(2):91-103.

13 [18] Chen Y-, Lewis JWL, Parigger C. Probability distribution of laser-induced 14 breakdown and ignition of ammonia. Journal of Quantitative Spectroscopy and 15 Radiative Transfer 2000 7/1;66(1):41-53. 


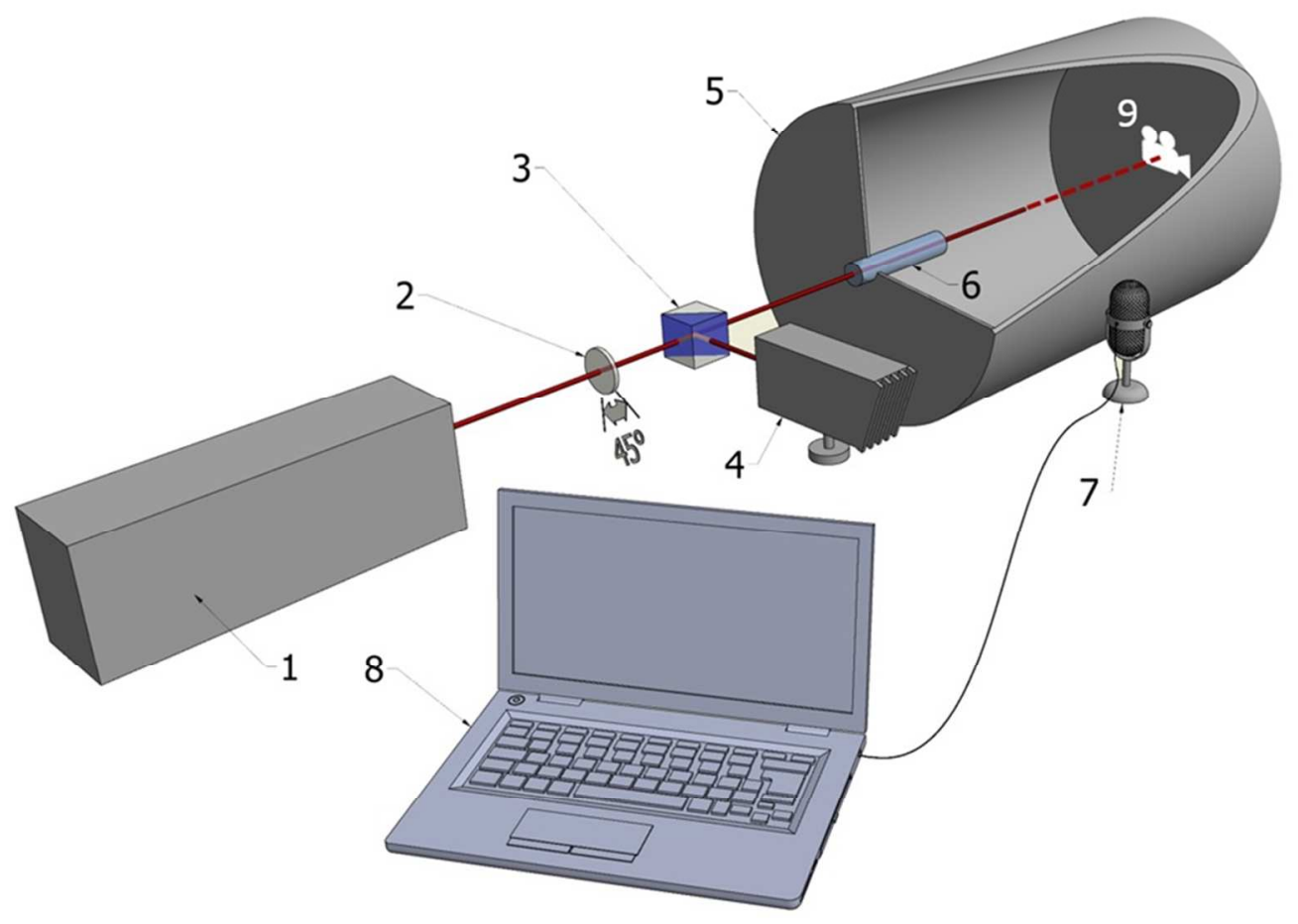

Figure 1: Experimental arrangement with 1) laser source, 2) 1/2 wave plate, 3) polarizing beam splitting cube, 4) beam dump, 5) combustion chamber, 6) laser igniter assembly, 7) microphone, 8) data acquisition computer and 9) camera.

$248 \times 180 \mathrm{~mm}$ (96 x 96 DPI) 


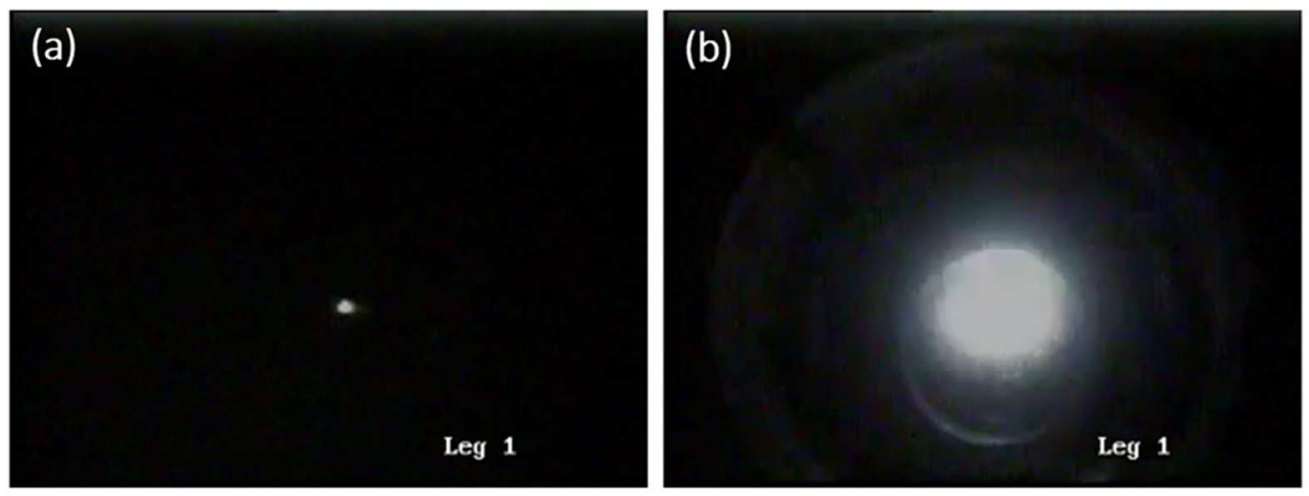

Figure 2: Camera feed showing (a) flashlamp operation and (b) laser induced breakdown (10 Hz repetition rate, $1064 \mathrm{~nm}$ wavelength).

$240 \times 89 \mathrm{~mm}(96 \times 96 \mathrm{DPI})$ 

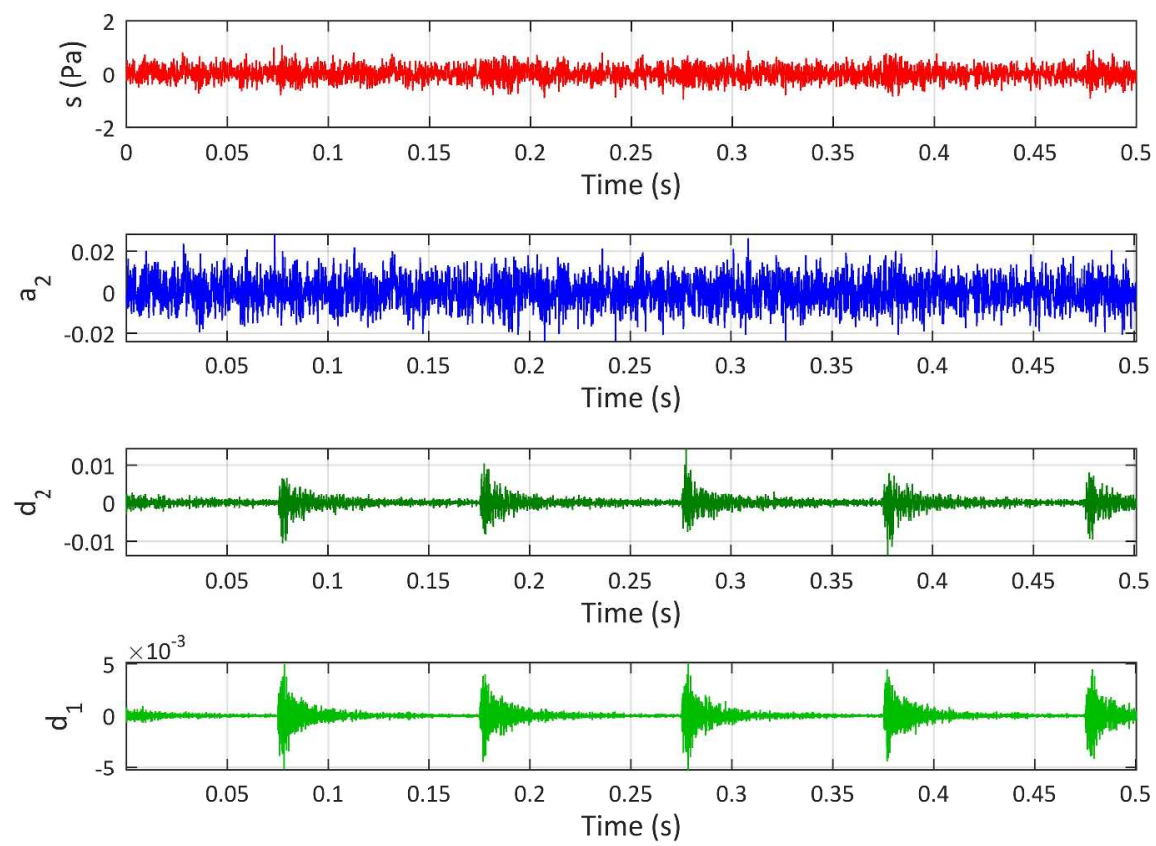

Figure 3: Two level Bior6.8 wavelet decomposition of raw acoustic sound pressure signal s with approximation coefficients a2, and detail coefficients $\mathrm{d} 1$ and $\mathrm{d} 2(21 \mathrm{~mJ}$ pulse energy, $10 \mathrm{~Hz}$ repetition rate, $1064 \mathrm{~nm}$ wavelength).

$2057 \times 1543 \mathrm{~mm}(72 \times 72 \mathrm{DPI})$ 

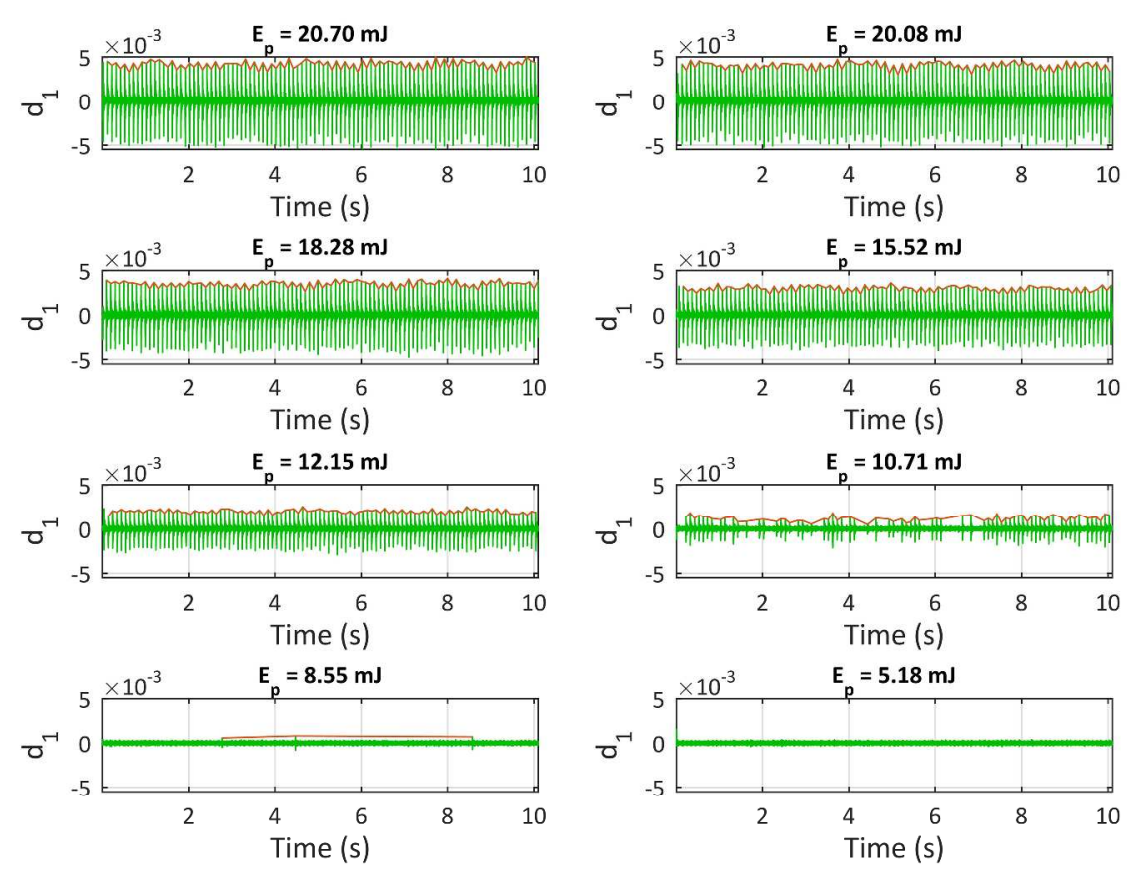

Figure 4: Representative outputs from the peak detection algorithm applied to the detail signal for a single $10 \mathrm{~s}$ audio recording (10 Hz repetition rate, $1064 \mathrm{~nm}$ wavelength).

$2057 \times 1543 \mathrm{~mm}(72 \times 72 \mathrm{DPI})$ 
(a)

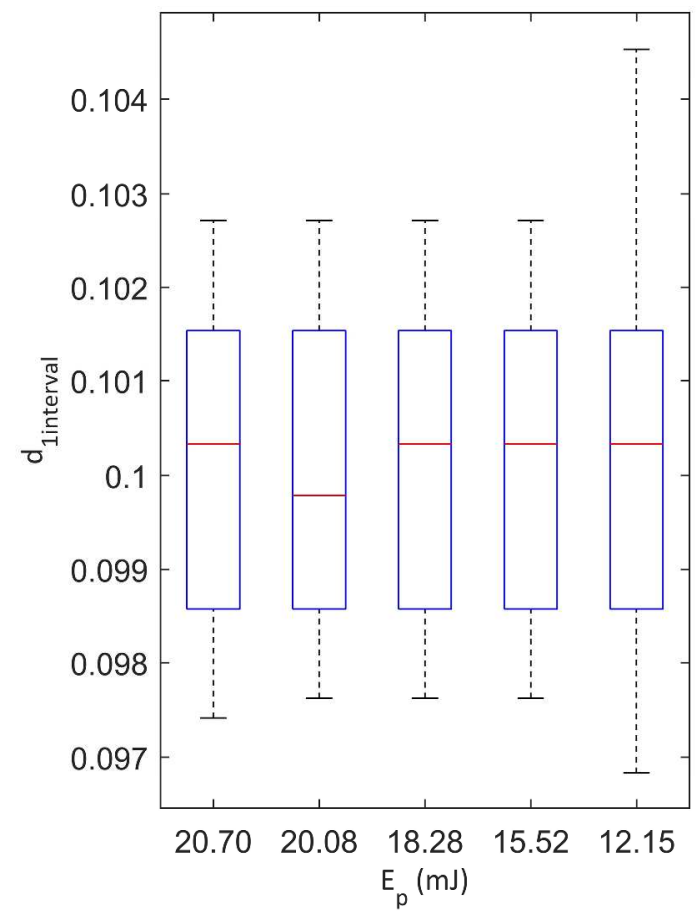

(b)

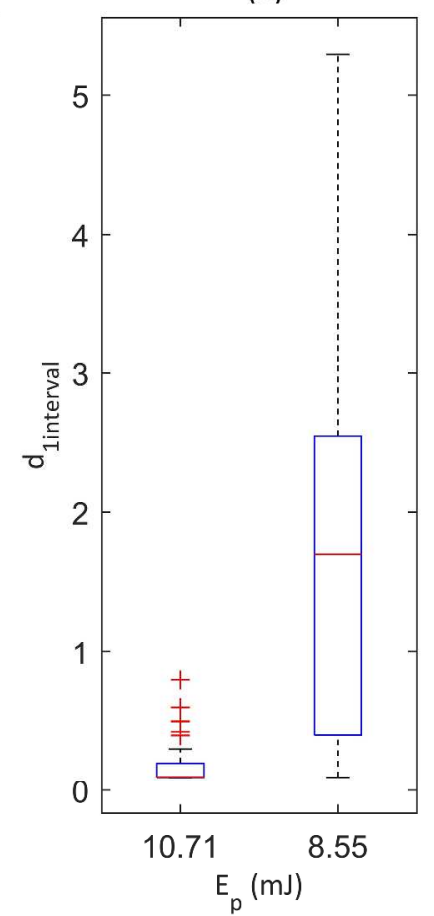

Figure 5: Box plot of the peak intervals (d1interval) from the peak detection algorithm applied to the detail signals in (a) the consistent laser induced breakdown region and (b) the inconsistent laser induced breakdown region (10 Hz repetition rate, $1064 \mathrm{~nm}$ wavelength).

$1543 \times 1157 \mathrm{~mm}(96 \times 96 \mathrm{DPI})$ 


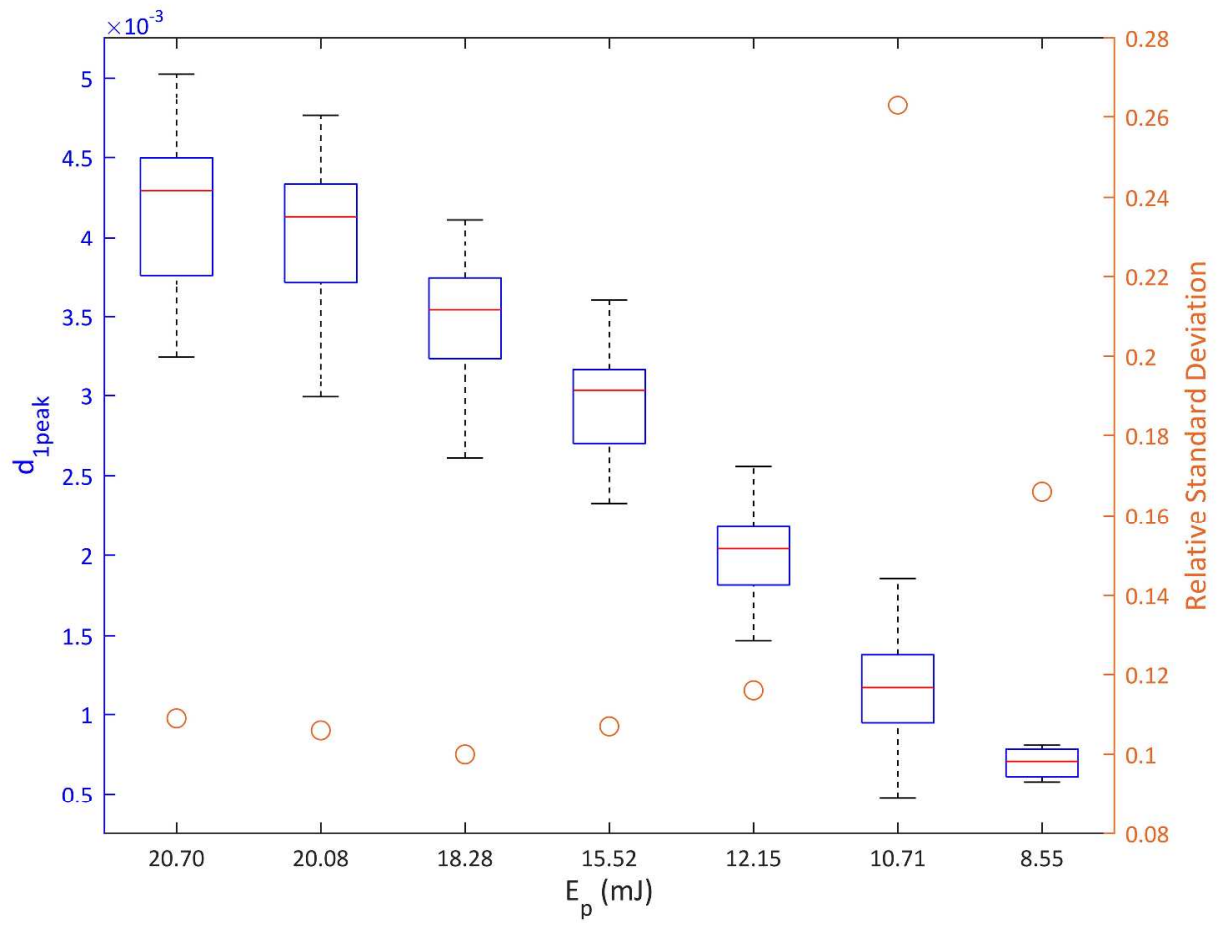

Figure 6: Box plot of absolute values of peak amplitude from the peak detection algorithm applied to the detail signal (d1peak) and their relative standard deviation (circles) as a function of incident pulse energy (10 $\mathrm{Hz}$ repetition rate, $1064 \mathrm{~nm}$ wavelength).

$2057 \times 1543 \mathrm{~mm}(72 \times 72 \mathrm{DPI})$ 